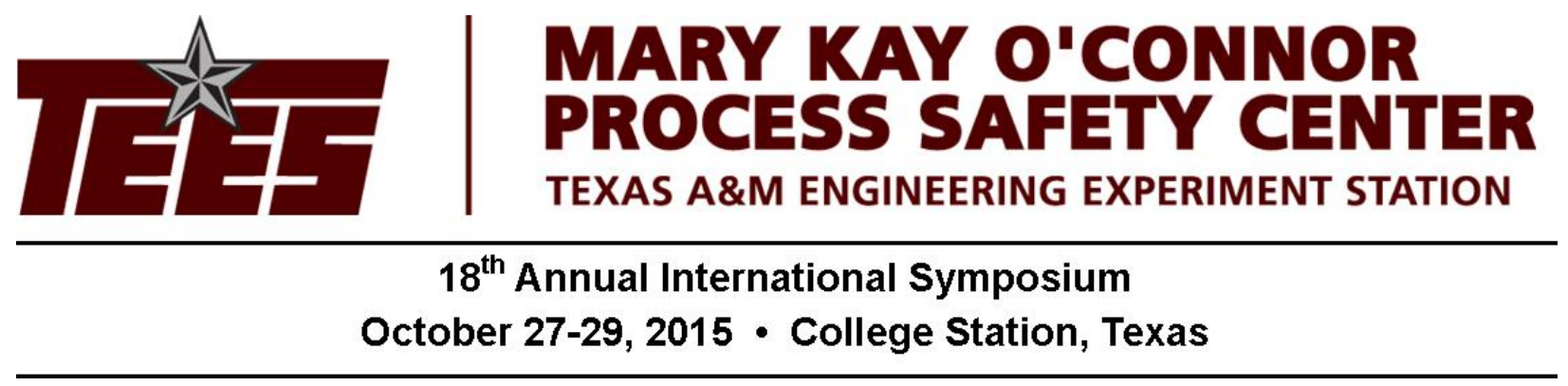

\title{
Ensuring Safe Facility Blowdown and Lessons Learnt from Incident Investigation
}

\author{
Dr. Praveen Lawrence \\ Dr. James Marriott \\ Dr. Apostolos Giovanoglou \\ Process Systems Enterprise Ltd \\ 26-28 Hammersmith Grove \\ London W6 7HA, England \\ p.lawrence@psenterprise.com
}

\begin{abstract}
A facility blowdown in the oil and gas industry is a safety critical operation; required to ensure the safe shut-down of processing facilities during a plant emergency. In a blowdown operation, the entire plant is isolated into a number of segments and then depressurized into the facility's flare system. The outcome of a blowdown event is dependent on the performance of a number of safety critical elements in the plant; these are designed to ensure the safe and successful depressurization of the plant without any conspicuous incidents. Blowdown events are inherently fast transient processes, reliant on a large number of valve opening and closing operations with rapid reduction in pressure and temperature. It is critical to ensure that the process is depressurized quickly enough (cf. API 521) but whilst respecting constraints such as minimum design metal temperatures, AIV/FIV limits in flare tailpipes and pressure constraints throughout the flare system. Blowdown incident investigations typically involve a critical review of the plant historian data and require dynamic simulation studies to adequately assess the event so as to determine whether the blowdown operation proceeded as planned, that no constraints were violated and to glean any process safety lessons that can be learnt.

In this talk, with reference to a number of recent events on oil and gas facilities, we explain how to carry out a comprehensive blowdown investigation. We will discuss a number of case studies from facilities in different parts of the world, including those that have shown operational deviations such as non-closure/partial closure ESD valves, delays in opening or non-opening of BDVs, non-operational check valves. Some of these deviations caused flare capacity issues in the plant, minimum design metal temperature (MDMT) violation risks to process drums, unexpected pressurization of process drums, flow reversal into low pressure segments causing near miss events, and other violations of recommended practice. We will explain how these
\end{abstract}


studies have helped understand the current state of the plant's safety barrier and have been used by operating companies to identify design and operational changes critical for safe plant operation. Finally we will discuss how these studies provided input to plant maintenance personnel in order to help prioritize maintenance activities and to make prudent maintenance investment.

\section{Introduction}

A blowdown operation is designed:

- to remove, in a controlled manner, all combustible hydrocarbons during an emergency situation or for a planned shutdown

- to reduce the risk of vessel or pipe rupture in the event of a fire

- to decrease the propensity for leaks by reducing process pressures

Typically prior to system depressurization a process plant is isolated into a number of independent blowdown segments. A full plant blowdown operation involves the simultaneous or staggered blowdown (depressurization) of all the pressurized gas (and/or in some cases liquid) in each segment by routing it to one or more flare tips for controlled combustion.

Though a blowdown operation is the last layer of safety defense in the plant, the operation may itself be hazardous and a number of factors must be considered. In particular a large amount of material must be disposed of in a relatively short time and it is necessary to ensure that there is sufficient flare discharge capacity without creating excessive pressure in the flare system or excessive velocities and vibration in the flare piping, over-filling knock-out (KO) drums, and exceeding radiation limits from the flare tip. The materials of construction of the facility must also be assessed carefully as extremely low temperatures can occur in both the process equipment and the flare system during a depressurization operation; particular consideration is required to avoid the risk of brittle fracture.

It is well documented (ref. 1) that a very significant number of safety incidents in oil and gas facilities take place during a transient operation in the plant such as blowdown, and so it is vital that such transient behavior is well understood.

\section{Model-based design and safety assessment}

The oil \& gas industry uses models and simulations to capture its understanding of its processing facilities and their safety systems. The models (and the data captured therein) typically form the basis of engineering decisions during the entire lifecycle of a plant and so thus form a critical safety component. For example, whether the flare system is adequately sized is assessed from model-based calculations, similarly relief valve sizes and adequacy of depressurization rate are all assessed using a model of the process and its safety system.

Most errors occur due to one of the following factors

- selection of an inappropriate model or set of assumptions

- incorrect model input data 
A particular issue is that during the life-cycle of a facility, typically 30-40 years, many changes to the facility will invariably be made and keeping the models up to date is challenging. Examples of facility changes that may affect the adequacy of the safety system include (i) changes in the amounts \& compositions of the oil, gas \& water that the facility processes (ii) facility extensions and design changes.

\section{Model-based blowdown event analysis:}

A blowdown event analysis is a critical review of a historic blowdown event; essentially checking that it proceeded safely and as expected. In this article, we will show that reviewing blowdown events using models is a vital to ensure that the facility's behavior is well understood.

The key inputs required to carry out a model-based assessment of a blowdown event include:

- Plant blowdown event data: Plant historians (e.g., OSIsoft's PI system ) capture a large amount of process data as a function of time, and data that are typically of interest for a blowdown event analysis are

o Process conditions at different locations within the process and flare network pressure, temperature, liquid levels

o Emergency shutdown (ESD) and blowdown valve (BDV) positions

It is also common that sensors may not be available in all the locations of interest and these limitations can be overcome when carrying out model based analysis.

- A fully coupled dynamic model of the blowdown segments and the flare network; this should accurately represent

0 the dynamics involved in the process blowdown segments and the flare network. Note that the dynamics of the flow in the flare network can lead to flow reversals at times into low pressure blowdown segments.

0 the staggering and staging of blowdown valves

0 the availability of flare tips some of which may be isolated by bursting discs

All the calculations reported in this paper were performed with models constructed in the gFLARE ${ }^{\circledR}$ Advanced Process Modeling software. It is common that plant operators have the flare network configuration captured in steady state flare network analysis tools (such as Aspen Flare System Analyzer - AspenTech ${ }^{\circledR}$ ) and automated tools are available to easily use this data to configure dynamic flare network models such as gFLARE.

The blowdown event analysis involves two stages of assessment. In stage 1, the plant historian data is processed (no models are required at this stage) to gather data that are required to define the blowdown event scenario to be studied using the models and also to answer some questions relating to the event:

- Determine the exact time at which blowdown event was initiated

- Check for any obvious deviations from the plant's blowdown procedure e.g., if all the BDVs actually opened during the blowdown operation

- Define the state of the plant at the start of the blowdown operation i.e., determine the pressure, temperature and liquid level in the process blowdown segments 
In stage 2, the data gathered from the review of the plant historian is applied to the full facility blowdown models and the simulations study results are analyzed and compared against the plant historian data to answer questions that relate to the behavior of the blowdown segments and the flare network. Typically, the following simulation results are compared against the plant historian data:

- Process blowdown and flare network pressures and fluid temperatures

- Liquid level process drums and flare KO drum

- Flow measurements at the flare stack and in any other location where measurement sensors are available

The above comparison provides very useful insight into the behavior of each of the blowdown segments and the flare network. Furthermore, one of the key advantages of model-based blowdown event analysis is that when deviations are found, the model can employed to understand the reasons for the deviation and rectify any inconsistencies in the understanding of the process thereby ensures that future engineering decisions are made using engineering data representative of the system.

In the next sections of this article, we give examples to further illustrate the benefit of modelbased blowdown event analysis.

\section{Case study 1: Flare network behavior during blowdown at an onshore Oil and Gas facility}

A recent blowdown event that took place at an onshore Oil and Gas facility (built in the 1980s), was analyzed to assess whether the behavior of the facility's safety system was correctly understood. Prior to depressurization, this facility is isolated into a number of blowdown segments ( 25 of them) and this is followed by a staged and staggered blowdown of the segments.

The existing dynamic full facility model (developed from an earlier project) was configured with the start conditions taken from the aforementioned PI historian and the blowdown event was simulated. Figure 1 shows a comparison of the calculated pressure at the Flare KO drum against the blowdown event data. 


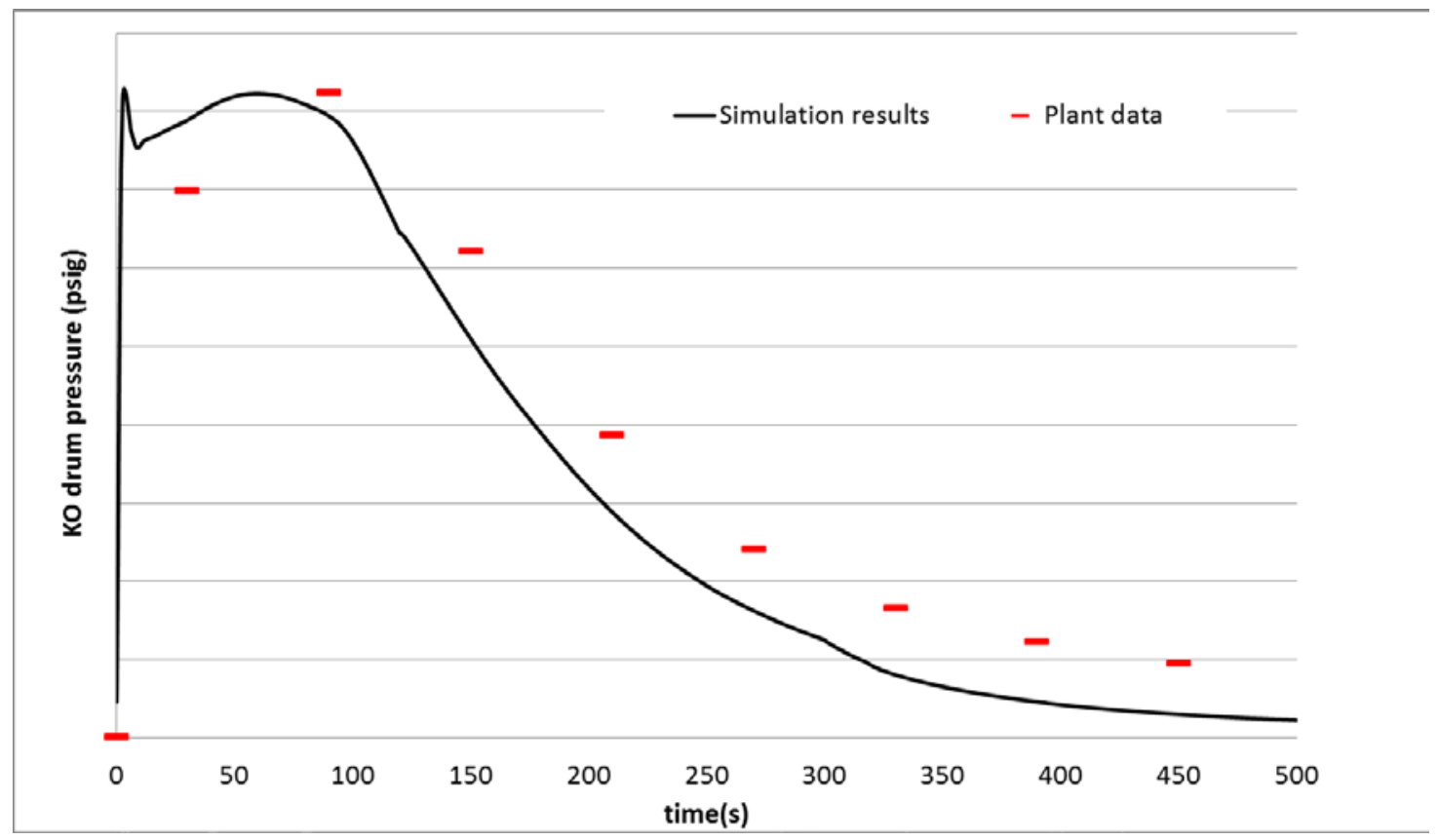

Figure 1: Comparison of flare KO Drum pressure between full facility simulation results and blowdown event data

Although, there were minor deviations from the plant blowdown procedure relating to nonopening of a BDV for one of the smaller blowdown segments and delay in the closure of ESDs, it was concluded that the model and the accompanying process understanding form a good basis for assessing the safety of the facility and for assessing flare performance KPIs like Mach number, Rhov2 values, pipe MDMT violations etc., in the flare piping to check for any design violations. These were checked using the model for the blowdown event starting conditions and also for the worst credible scenarios defined for the facility.

\section{Case study 2: Flare network behavior during blowdown at an FPSO facility}

A blowdown event took place at a recently built FPSO facility. The blowdown operation involved staggering of the various blowdown sources in two steps. Like most facilities, the sensors in the flare network system were limited and those present were concentrated around the flare KO drum and flare stack; so only limited data was available to assess the performance of the flare network.

As in Case 1, a dynamic full facility model (developed from an earlier project) was configured with the start conditions taken from the facility's historian. The model was based on the engineering data maintained for the facility

The study showed that the calculated peak pressure at the sensor location (KO drum) was roughly $50 \%$ of the measured plant data (see Figure 2) and this inconsistency raised questions on the accuracy of the input specifications to the model. The inconsistency could be at one or more of the following reasons: 
- More material entering the flare network from blowdown sources not accounted for

- Inaccurate understanding of the blowdown segment volumes

- Errors in the datasheets for RO or valve datasheets and flare tip

- Incorrect understanding of the flare pipe sizes

A number of what-if sensitivity studies on flare tip performance and engineering data checks revealed possible causes the flare network model configured to have a flare stack of 32" diameter while the installed flare stack was found to be only 24" in diameter and when this specification error was corrected the performance of the flare network was well represented by the simulations (see Figure 2).

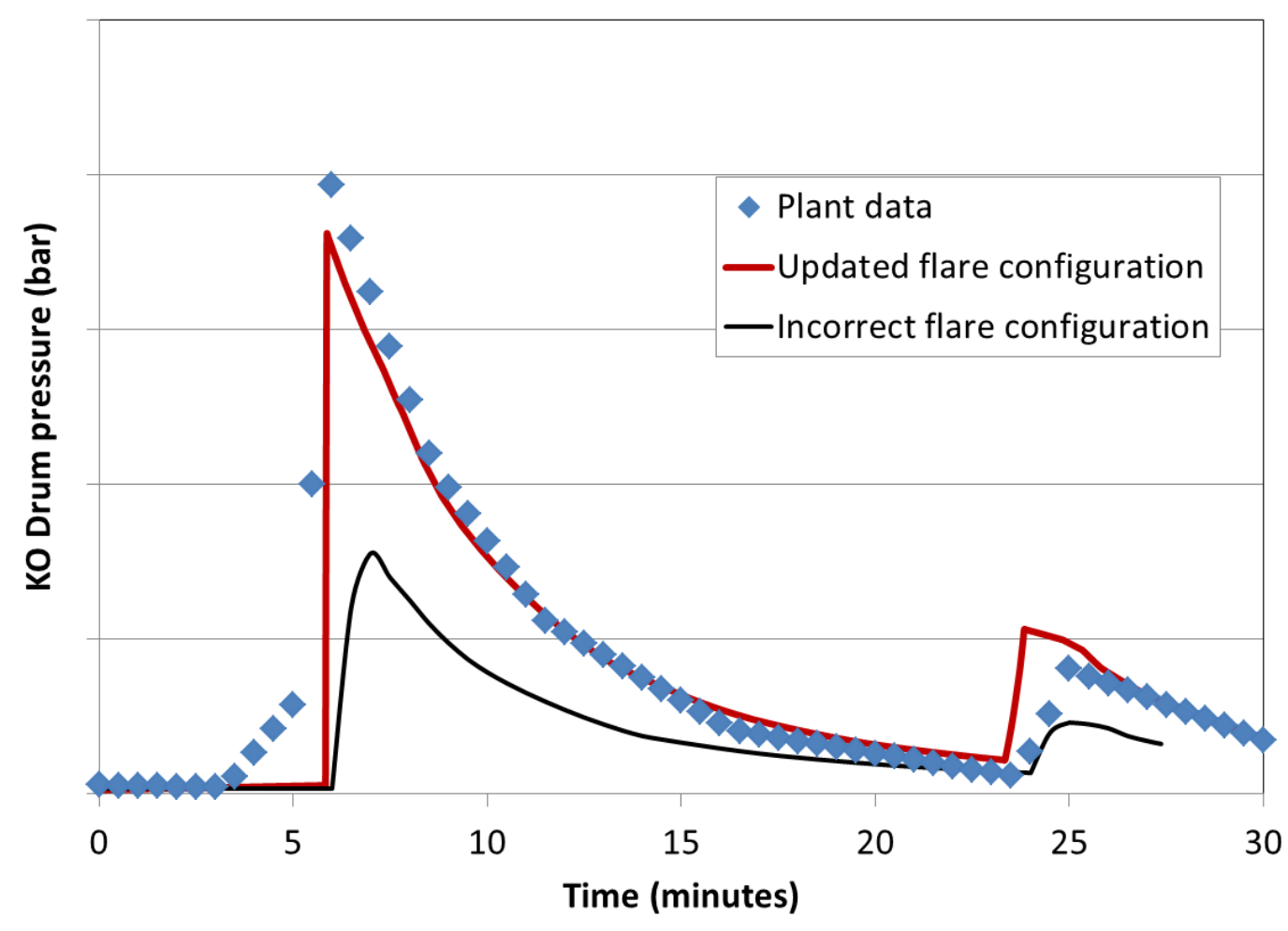

Figure 2: Comparison of plant blowdown event data against two simulation results (Incorrect and correct flare network model configuration)

In this case, as all the process design studies were carried out using an incorrect flare stack diameter (32" instead of 24") specification and as the design phase calculations were not conservative, the worst case design scenarios were repeated again with the updated flare stack diameter specification and checked for any design mitigation requirements.

\section{Case study 3: Process KO drums blowdown segment behavior at a natural gas processing facility}

A recent blowdown event that took place at an onshore natural gas processing facility (built in the 1980s) was analyzed to assess whether the process and its safety system were correctly understood. As part of the blowdown procedure, the plant was isolated into 6 blowdown 
segments of which 5 of the segment BDVs were staged. One of the blowdown segments involved a process knock out (KO) drum with heat integrated process coolers around the drum. The blowdown segment had two blowdown valves - to relieve vapor and to drain the liquid inventory in the drum.

The blowdown event data showed that the segment depressurized at a considerably slower rate than expected and liquid inventory was present in the KO Drum for ca. 45 minutes, the segment was investigated to understand the reasons for the slow depressurization rate.

As per design, the liquid blowdown valve must completely drain the liquid in the KO Drum in a short time ( $<3$ minutes) and would be followed by vapor relieving through this valve to ensure that the segment is depressurized as per design. Clearly, during the blowdown event, the liquid took a considerably long time to depressurize (in ca. 45 minutes) and this reduced the vapor relief rate from the system which caused the blowdown segment pressure to be above 200psig for more than an hour (after start of blowdown).

Sensitivity studies on the liquid drain rate showed that the blowdown valve opened only partially and restricted the liquid drain rate resulting in the deviation in the performance of the blowdown segment. At the next shut-down, plant maintenance personnel were given (along with a number of other facility-wide actions arising from the study) a number of issues to check relating to this blowdown segment. From the maintenance assessment, it was confirmed that asphalt deposition at the valve was the likely cause for this behavior. Figure 3 shows a comparison of the liquid drain rate and segment pressure from the plant data against one of the sensitivity studies that correctly represented the system behavior.

Thus, in this case, though the process understanding about the system was correctly captured in the models, the deviation in system behavior was caused by operational issues and the model could help pinpoint the reasons for the deviation, which was later rectified in the plant following a guided maintenance review.

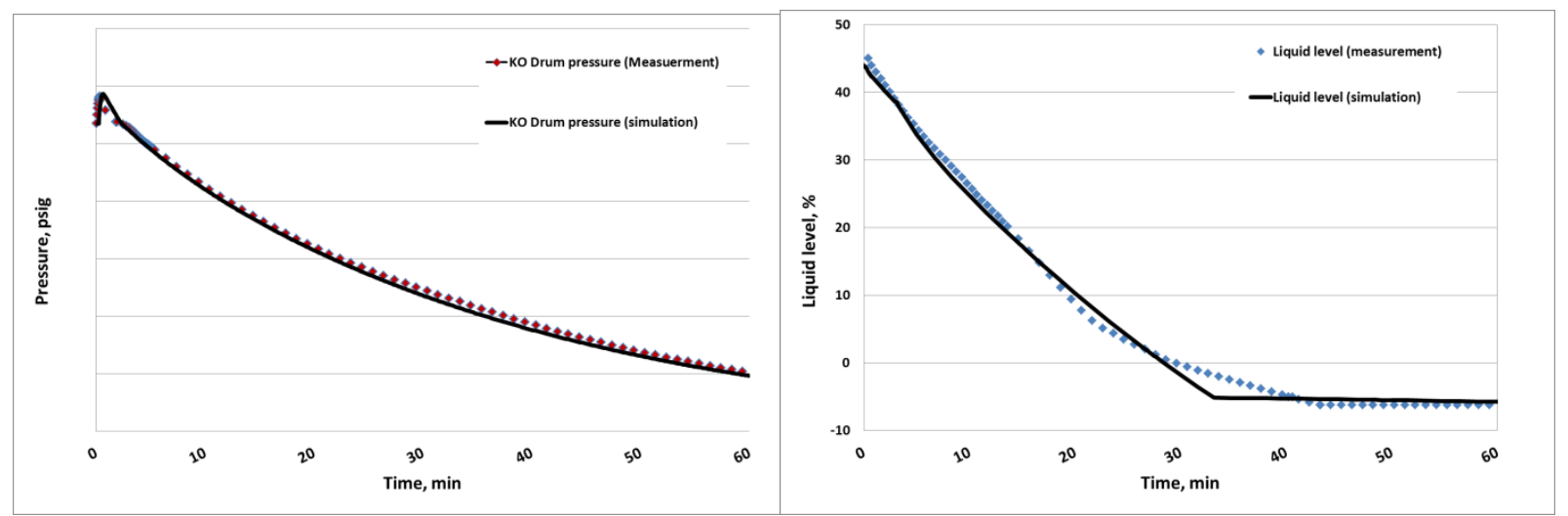

Figure 3: Plant data vs. simulation (a) Comparison of liquid level in the KO drum (b) KO drum pressure. Correction for the valve opening resulted also in a good agreement for calculated pressure 


\section{Conclusions}

Blowdown/flare systems typically provide the last line of defense to prevent loss of primary containment or escalation when a loss of containment or fire has occurred. Given this critical function ensuring that they behave as expected is a critical requirement for any facility.

The authors of this paper have used model-based analysis to assess many blowdown events on behalf of the operators of offshore and onshore oil \& gas processing facilities. In some facilities only minor issues are found but in others significant issues have been identified. Typical problems that are identified include

- Deviations from blowdown procedure, e.g., slow valve closure rate of ESD valves resulting in unexpected pressure equalization within process segments

- Inaccurate representation of process thermodynamics resulting in deviations in the amount of liquid and vapor in the flare system.

- Deviations in depressurization rate either due to incorrect blowdown segment volume or incorrect BDV/RO specifications

- Incorrect flare piping size representation in the model or P\&IDs/isometrics resulting in over or under prediction of flare pressure

- BDV and flare system operation affected due to blockage due to foreign object or hydrates

Finally it is noted that whilst considerable attention is given to the steady operation of a facility; analysis of its transient behavior during depressurization is not always given the attention it needs.

\section{Reference:}

1) “Guidelines for the safe \& optimum design of hydrocarbon pressure relief \& blowdown systems” Institute of Petroleum, London, 2001 